\title{
High-efficiency low-crosstalk dielectric metasurfaces of mid-wave infrared focal plane arrays
}

Onur Akın, and Hilmi Volkan Demir

Citation: Appl. Phys. Lett. 110, 143106 (2017); doi: 10.1063/1.4979664

View online: https://doi.org/10.1063/1.4979664

View Table of Contents: http://aip.scitation.org/toc/apl/110/14

Published by the American Institute of Physics

\section{Articles you may be interested in}

Electrically tunable all-dielectric optical metasurfaces based on liquid crystals

Applied Physics Letters 110, 071109 (2017); 10.1063/1.4976504

Reconfigurable conversions of reflection, transmission, and polarization states using active metasurface

Applied Physics Letters 110, 121901 (2017); 10.1063/1.4979033

Ultra-compact visible chiral spectrometer with meta-lenses

APL Photonics 2, 036103 (2017); 10.1063/1.4974259

Integrating polarization conversion and nearly perfect absorption with multifunctional metasurfaces

Applied Physics Letters 110, 171903 (2017); 10.1063/1.4982240

Adaptable metasurface for dynamic anomalous reflection

Applied Physics Letters 110, 201904 (2017); 10.1063/1.4983782

Invited Article: Broadband highly efficient dielectric metadevices for polarization control

APL Photonics 1, 030801 (2016); 10.1063/1.4949007

\section{PHYSICS TODAY}

MANAGER'S GUIDE

WHITEPAPERS
READ NOW

PRESENTED BY

Accelerate R\&D with
Multiphysics Simulation 


\title{
High-efficiency low-crosstalk dielectric metasurfaces of mid-wave infrared focal plane arrays
}

\author{
Onur Akın ${ }^{1, a)}$ and Hilmi Volkan Demir ${ }^{1,2, a)}$ \\ ${ }^{1}$ Department of Electrical and Electronics, Department of Physics, and UNAM-Institute of Material Science \\ and Nanotechnology, Bilkent University, TR-06800 Ankara, Turkey \\ ${ }^{2}$ LUMINOUS! Centre of Excellence for Semiconductor Lighting and Displays, School of Electrical and \\ Electronic Engineering, Nanyang Technological University, 50 Nanyang Avenue, 639798 Singapore
}

(Received 30 December 2016; accepted 22 March 2017; published online 4 April 2017)

\begin{abstract}
High-resolution compact-size focal plane arrays (FPAs) suffer the fundamental geometrical tradeoff between the optical resolution (pixel size miniaturization) and the optical crosstalk (spillover of neighboring pixel focusing). For FPAs, our previously reported metallic metasurfaces reached an unprecedented level of crosstalk suppression. However, practical utilization of these metallic microlens arrays has proved to be intrinsically limited due to the low device efficiency (of the order of 0.10) resulting from the fundamental absorption losses of metals and their cross-polarization scheme. Exceeding this limit, here we show highly efficient microlens designs enabled by dielectric metasurfaces for mid-wave infrared (MWIR) operation. These dielectric MWIR FPAs allow for a substantially high device efficiency over 0.80 without compromising the optical crosstalk performance. Systematically studying dielectric nanoantennas of silicon nanodisks that do not dictate the cross-polarization scheme using full-wave solutions, we found that the optical crosstalk is suppressed to low levels $\leq 3.0 \%$ while sustaining the high efficiency. A figure-of-merit (FoM) defined for the device performance as the focusing efficiency per optical crosstalk times the f-number achieves 84 , which is superior to all other types of MWIR FPAs reported to date, all falling below a maximum FoM of 70. These findings indicate that the proposed approach can pave the way for the practical usage of metasurface microlens arrays in MWIR. Published by AIP Publishing.

[http://dx.doi.org/10.1063/1.4979664]
\end{abstract}

Optical crosstalk is a critical parameter of mid-wave infrared (MWIR) focal plane arrays (FPAs), which affects the performance of electro-optical systems used in detection of small objects having a low signal-to-noise ratio (SNR). The fundamental tradeoff between the optical crosstalk and the pixel pitch size stands as a major obstacle to the development of next-generation MWIR FPAs with a higher resolution and a smaller size. Moreover, as the f-number of conventional MWIR FPAs increases (for reducing optical aberrations), so does the optical crosstalk. ${ }^{1}$ To date, for removing this obstacle, integration of different types of microlens arrays to MWIR FPAs has been reported. ${ }^{1,2}$ Refractive type microlens arrays narrowed the spot size of the incoming beam but suffered from the emergence of diffraction spots and was not able to improve the optical crosstalk. ${ }^{1}$ In a previous work reported by our group, metallic metasurface microlens arrays were shown to suppress the optical crosstalk to less than $1 \%$ but the focusing efficiency, which is the ratio of the focused photon energy to that of the incident photon energy, was too low $(<11 \%)$ to make these devices practical. $^{2}$ Although transmission-mode metallic metasurfaces have been studied extensively and they offer promising features including broadband functionality, the device efficiency has become a common problem. ${ }^{3-12}$ Here, high absorption losses and cross-polarized focusing schemes are the main reasons for this typical drawback.

\footnotetext{
a)Electronic addresses: oakin@bilkent.edu.tr and volkan@stanfordalumni.org
}

Recently, dielectric nanoantennas have been used as building blocks of high efficiency metasurfaces. ${ }^{13-28}$ Metalenses with a diffraction-limited focusing efficiency up to $86 \%$ were realized using $\mathrm{TiO}_{2}$ nanofins ${ }^{29}$ while subwavelength thick lenses with a focusing efficiency reaching $82 \%$ were realized using silicon posts. ${ }^{21}$ Although the focusing efficiencies were promising, the designs were for the visible and near infrared while the $\mathrm{TiO}_{2}$ nanofins and the silicon posts were oversized for designing an MWIR microlens. Using Si nanodisks having a relatively smaller size, beam shaping was studied but the device efficiency was slightly lower and the design was for the near infrared. ${ }^{19}$ However, dielectric metasurfaces of MWIR microlens arrays were not previously studied. Their great potential for FPAs, which is technologically critical for the next-generation, high-density, high-SNR FPAs, has not been exploited, and their device performance in MWIR remains unknown.

In this study, different from our previous work and the recent reports on metasurfaces, we address the optical crosstalk and efficiency problems in developing dielectric metasurfaces of MWIR FPAs. For increasing the device efficiency, here we propose and show a dielectric microlens array integrated to MWIR FPAs. While improving the efficiency dramatically, we fulfill at the same time the superior optical crosstalk performance of metallic metasurfaces of MWIR FPAs that suffer undesirably high, fundamental absorptive loss. Another key consideration taken into account here is to prevent the increase in the optical crosstalk due to the increase in the f-number, thereby paving the way 
for higher f-number MWIR FPAs with a low optical crosstalk.

For achieving all these goals, dielectric metasurfaces of MWIR FPAs based on Si nanodisks were designed. Here, we first focused on the specific design of a set of Si nanodisks (see Fig. 1(a)). Then, the phase shift profile of the microlens arrays was designed for optimizing the device performance in the detection of small objects having a low SNR by increasing the object signal and decreasing the noise. To increase the object signal, the focused optical energy in the central pixel was required to be maximized by increasing the focusing efficiency of the microlens array. To decrease the noise, the background signal and the optical energy spilling over into the neighbor pixels were required to be minimized by increasing the f-number and decreasing the optical crosstalk, respectively. For describing this optimized device performance, a figure-of-merit (FoM) was defined as the ratio of the product of the focusing efficiency and the f-number to the optical crosstalk. Following the design, the microlens array was realized by placing the nanodisks (see Figs. 1(b) and 1(c)). Finally, focusing efficiency and optical crosstalk performance were comparatively studied considering different types of microlens arrays and conventional MWIR FPAs reported to date. As a proof-of-concept demonstrator, we showed $\mathrm{a} \geq 80 \%$ efficiency dielectric microlens array scheme that has the highest performance in comparison to all previous MWIR FPAs, without a significant compromise of the optical crosstalk performance $(\leq 3 \%)$. The proposed dielectric metasurface FPAs reaching a FoM of 84 exceeded all other classes of FPAs, obtaining a maximum FoM of 70.

For designing the set of Si nanodisks, the initial parameters for diameter and height were computed using the resonance and transmission conditions. For the MWIR band (3-to-5 $\mu \mathrm{m}$ ), the diameter was varied from approximately 1000 to $1800 \mathrm{~nm}$ while the height was half of the former values. However, keeping the diameter-to-height ratio constant for maximizing the transmission efficiency resulted in limited phase shift coverage. Therefore, the diameter was also changed for fixed height values during simulations. The unit cell in which the nanodisk was placed was a square with the edge length varying from 1500 to $2500 \mathrm{~nm}$. While varying the edge length, we considered the size tradeoff between the mutual coupling and the scattering efficiency. For modeling and simulating these Si nanodisks, full-wave simulations were performed using the Lumerical finite difference time domain (FDTD) solver. Each nanodisk was placed at the center of a simulation region and surrounded by a homogeneous medium. A monochromatic plane wave source was used to excite the nanodisk and a near-field monitor was placed to record the near-field data which were transformed to a far-field. As boundary conditions, periodic boundary conditions were used along the axial directions and the perfectly matched layer (PML) boundary condition was imposed in the normal direction. After a series of simulation runs, an optimized $\mathrm{Si}$ nanodisk set was designed for the wavelength of $3.2 \mu \mathrm{m}$ and for the homogeneous medium

(a) Scattering amplitude and phase shift responses of nanodiscs

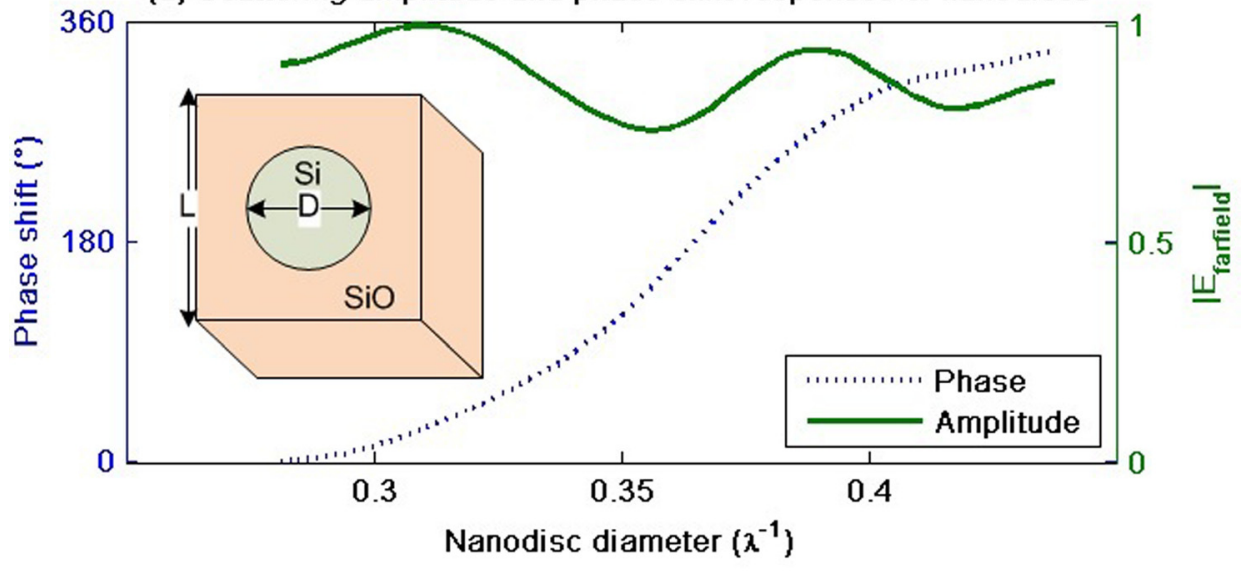

(b) Continuous phase shift profile

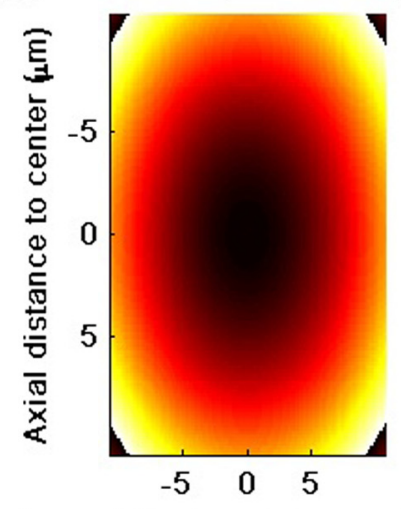

Lateral distance to center $(\mu \mathrm{m})$ (c) Discretized phase shift profile
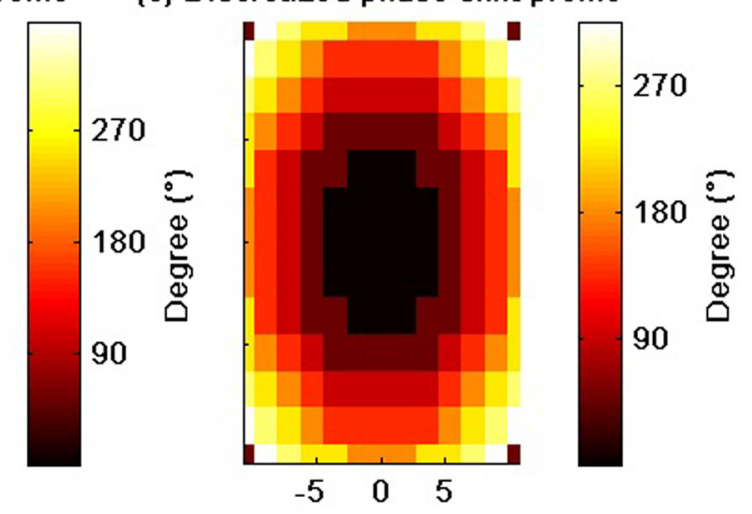

Lateral distance to center $(\mu \mathrm{m})$
FIG. 1. (a) Scattering amplitude and phase shift responses of Si nanodisks that cover the 0 -to- $2 \pi$ phase shift span with a highly uniform amplitude response (simulated geometry of the $\mathrm{Si}$ nanodisk model is shown in the inset). (b) Ideal (continuous) phase profile that should be imparted by a single microlens in the microlens array having a pitch length of $20 \mu \mathrm{m}$. (c) Discretization of the ideal phase profile for realization with Si nanodisks inside unit cells having an edge length of $1800 \mathrm{~nm}$. 
refractive index of 1.42. The resulting phase shift and amplitude responses of these Si nanodisks with specified diameters are given in Table I (with the height fixed at $550 \mathrm{~nm}$ ). The main advantage gained by using $\mathrm{Si}$ nanodisks was the dramatically increased device efficiency. The unit cells containing the metallic nanoantennas scattered cross-polarized light with an efficiency of $11 \%$ while the unit cells containing the Si nanodisks transmitted with an efficiency more than $65 \%$ and even reaching $90 \%$ for the diameter of $1020 \mathrm{~nm}$ (corresponding to the diameter-to-height ratio of the Kerkers condition at which the electric and magnetic dipoles spectrally overlap, allowing for almost unity transmission ${ }^{30}$ ).

The ideal phase profile of a metalens is given in Eq. (1). To reduce the optical crosstalk while keeping the f-number (f/\#) larger than a reasonably high value (1.5), a design methodology based on the restriction of a parameter of Eq. (1) was developed. According to this methodology, the focal length variable was limited to a certain range of values that provide a phase shift of at least $\pi$ radians between the center and the edge of a microlens in the microlens array while keeping a ratio that is greater than the threshold of 1.5 between itself and the aperture size of the microlens. This methodology was used in designing our dielectric metasurfaces of MWIR FPAs having focal lengths tuned from 15 to $90 \mu \mathrm{m}$ and aperture sizes varied from 20 to $30 \mu \mathrm{m}$ depending on the value of the focal length parameter.

For realizing the designed profile with Si nanodisks, this profile must be discretized (see Fig. 2(a)). After realization, full-wave simulations were performed. In Fig. 2(b), far-field distribution of light (the same for TM and TE polarizations) scattered from the central microlens of the optimized design is presented. As seen in Fig. 2(b), unlike the metallic metasurfaces that require the cross-polarization scheme to function, the dielectric metasurface can focus both polarizations of light. When the source wavelength was changed to $3.5 \mu \mathrm{m}$, the focusing behavior of the microlens array was disturbed (see Fig. 2(c)). This narrowband response limits the usage of dielectric microlens arrays in the full spectrum of the MWIR band. This is a drawback of the dielectric metasurfaces when compared to the metallic metasurfaces (also designed using Eq. (1)) that can work over a broader range of wavelengths. Far-field light distribution of all the microlenses is given in Fig. 2(d)

$$
\theta=\frac{2 \pi}{\lambda}\left(\sqrt{x^{2}+y^{2}+f^{2}}-f\right),
$$

TABLE I. Far-field responses of the designed Si nanodisks.

\begin{tabular}{lccc}
\hline \hline Nanodisk No & Phase shift (deg) & Scattering amp. & Diameter (nm) \\
\hline 1 & 35 & 0.94 & 1240 \\
2 & 79 & 0.82 & 1320 \\
3 & 125 & 0.92 & 900 \\
4 & 167 & 0.98 & 1020 \\
5 & 214 & 0.82 & 1090 \\
6 & 256 & 0.77 & 1130 \\
7 & 295 & 0.77 & 1160 \\
8 & 347 & 0.87 & 1200 \\
\hline \hline
\end{tabular}

$$
\text { Crosstalk }=100 \times \frac{\int_{A_{\text {neighbor }}} \operatorname{PSF}(x, y) d A}{\int_{A_{\text {central }}} \operatorname{PSF}(x, y) d A} .
$$

For FPAs, the point spread function (PSF) characterizes the optical crosstalk, ${ }^{1}$ which can be defined as the ratio of the corresponding PSF distributions inside the neighbor and central pixels (Eq. (2)). The optical crosstalk values of designed microlens arrays were compared with other types of MWIR FPAs. Although the dielectric metasurfaces have a lower optical crosstalk $(\leq 3 \%)$ than the refractive microlens arrays and the conventional MWIR FPAs, the metallic metasurfaces have the lowest optical crosstalk $(\leq 2 \%)$ for $\mathrm{f}$ numbers greater than 1.5 . The primary reason for the slightly degraded optical crosstalk performance was the undersampling of the phase profile due to a greater unit cell size of the dielectric metasurfaces (more than twice of the metallic unit cells). Because of this undersampling, the overlap between the discretized response and the ideal response was decreased. This overlap reduction slightly increased the unwanted scattering side lobes that increase the optical crosstalk. When the focusing efficiency is considered, the dielectric metasurfaces $(\geq 80 \%)$ outperform the metallic ones $(\leq 11 \%)$ by a substantial factor of 8 , enabling the practical usage of dielectric metasurface microlens arrays. Main reasons for this improvement are the lack of the crosspolarization scheme and the removal of the intrinsic absorption losses occurring in the metallic nanoantennas.

For detecting low SNR objects, the photons irradiated from the object should be collected as much as possible. Thus, the focusing efficiency of microlens arrays should be as high as possible. More importantly, these photons should be collected by the right pixel by minimizing the optical crosstalk for improving the spatial SNR. However, conventional MWIR FPAs with a relatively lower optical crosstalk have small f-numbers, which is disadvantageous for reducing optical aberrations. ${ }^{1}$ Therefore, the overall device performance is directly proportional to the focusing efficiency and the f-number while inversely proportional to the optical crosstalk. For this reason, an FoM is defined as follows:

$$
F o M=\frac{\mathrm{f} / \# \times \xi_{\text {efficiency }}}{\text { crosstalk }} .
$$

While designing and optimizing our dielectric metasurfaces, we aimed to maximize this FoM (Eq. (3)). Fig. 3 shows the FoM comparison of different types of MWIR FPAs. Despite achieving an excellent optical crosstalk, metallic metasurfaces of MWIR FPAs have the lowest FoM values due to their very poor focusing efficiency. Refractive microlens arrays of MWIR FPAs do not improve either the optical crosstalk or the f-number and hence the FoM. The arrow in Fig. 3 shows the performance evaluation of our proposed metasurfaces following our methodology which reaches the highest FoM after achieving low optical crosstalk values (with higher f-numbers) compared to the reflective microlens array and conventional MWIR FPAs while having a dramatically improved focusing efficiency compared to the metallic metasurfaces. However, metasurface microlens 
(a) Realization of Microlens

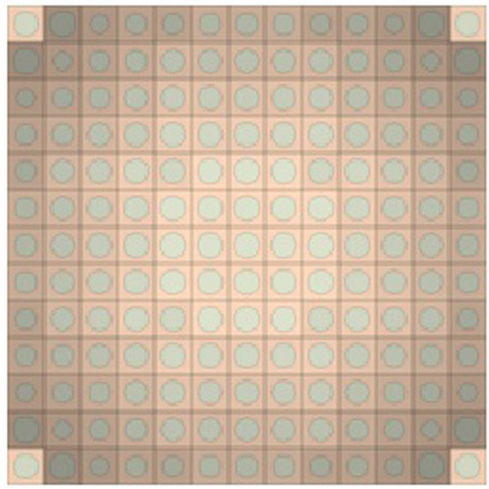

(c) Microlens focusing (off-resonance)

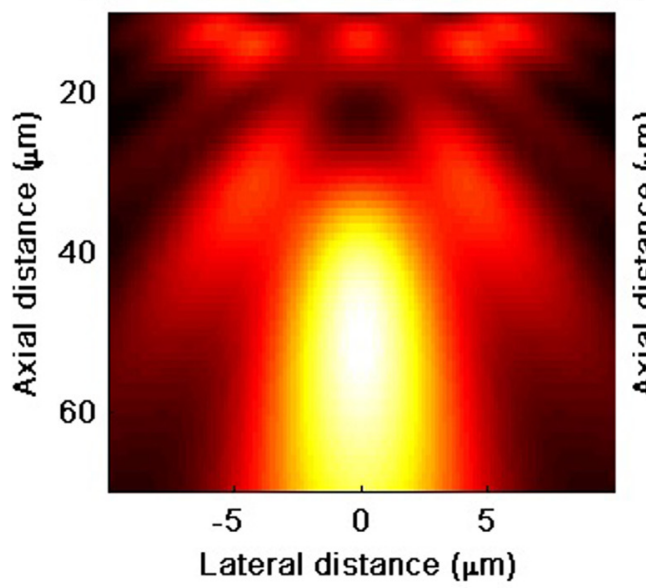

(b) Microlens focusing (TM and TE)

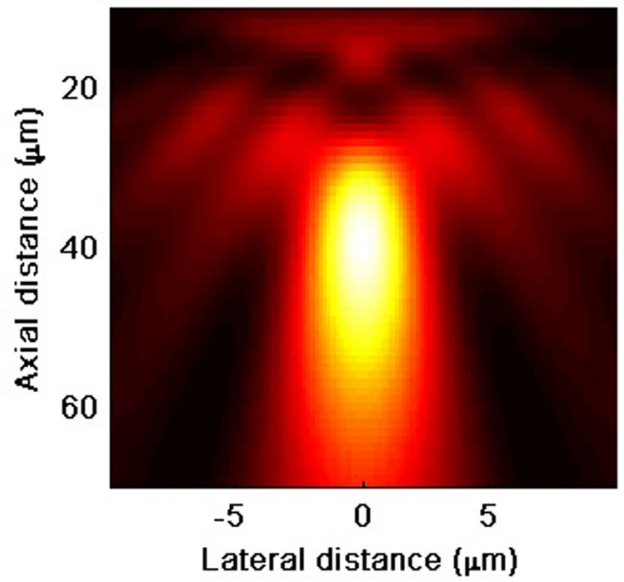

(d) Microlens array focusing

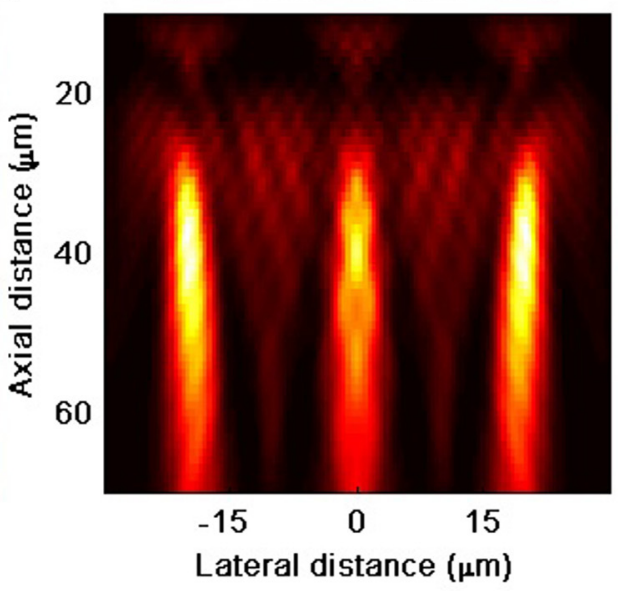

FIG. 2. (a) Realization of the optimized design's central microlens by $\mathrm{Si}$ nanodisks (the color of unit cells is graded within constant phase regions). (b) Far-field intensity distribution of light focused by the central microlens of the optimized design when excited with either TM or TE polarized light. (c) Far-field intensity distribution of light focused by the central microlens of the optimized design for the offresonance wavelength of $3.5 \mu \mathrm{m}$ which is different from the on-resonance design wavelength. (d) Far-field intensity distribution of light focused by different pixels of the optimized microlens array design. array integration can have a slightly degraded FoM performance in practice due to imperfect phase realization resulting from possible fabrication imperfections in sizes of silicon nanodisks.

In conclusion, we have proposed and demonstrated efficient, low-crosstalk high-SNR microlens array integrated

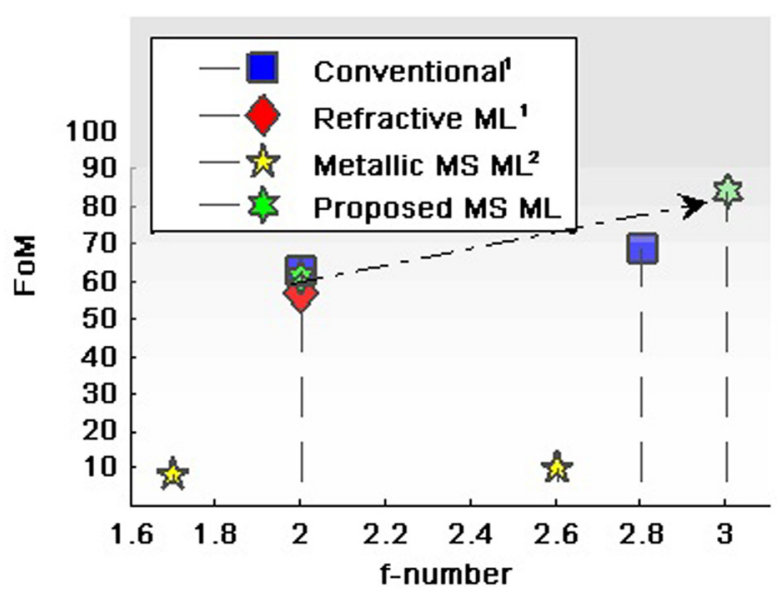

FIG. 3. FoM comparisons of different types of MWIR FPAs, showing the superior performance of the proposed (dielectric) metasurface microlensed FPAs (green hexagram marker) over the conventional FPAs ${ }^{1}$ (blue square markers suffering from the higher optical crosstalk), the refractive microlensed FPAs $^{1}$ (red circle marker suffering from diffraction noise), and the metallic metasurface microlensed FPAs ${ }^{2}$ (yellow pentagram marker suffering from very poor transmission performance).
MWIR FPA designs based on dielectric metasurfaces that can be used in the MWIR region of the infrared spectrum. We confirmed the expected behavior of these designs, analyzed the focusing efficiency and crosstalk performances by performing full-wave simulations and compared these results with those of the microlens arrays based on metallic metasurfaces and other types of microlens arrays and conventional FPAs. We showed that high focusing efficiency (over 80\%) can be obtained while achieving the best FoM (at a level of 84) surpassing the FoM of all other types of MWIR FPAs and preserving the ultralow optical crosstalk (at a level of $2.6 \%$ ), which is still superior to refractive microlens arrays without the inclusion of the mesa-isolation technique. The proposed MWIR microlens arrays can be used in FPAs possibly to detect and track small and dim objects at unprecedented low rates of false alarm, which will be the future step of this work.

${ }^{1}$ Y. Li, Z.-H. Ye, W.-D. Hu, W. Lei, Y.-L. Gao, K. He, H. Hua, P. Zhang, Y.-Y. Chen, C. Lin et al., J. Electron. Mater. 43, 2879 (2014).

${ }^{2}$ O. Ak in and H. V. Demir, Opt. Express 23, 27020 (2015).

${ }^{3}$ P.-C. Li, Y. Zhao, A. Alu, and T. Y. Edward, Appl. Phys. Lett. 99, 221106 (2011).

${ }^{4}$ F. Aieta, P. Genevet, M. A. Kats, N. Yu, R. Blanchard, Z. Gaburro, and F. Capasso, Nano Lett. 12, 4932 (2012).

${ }^{5}$ X. Ni, S. Ishii, A. V. Kildishev, and V. M. Shalaev, Light: Sci. Appl. 2, e72 (2013).

${ }^{6}$ F. Aieta, P. Genevet, M. Kats, and F. Capasso, Opt. Express 21, 31530 (2013). 
${ }^{7}$ D. Hu, X. Wang, S. Feng, J. Ye, W. Sun, Q. Kan, P. J. Klar, and Y. Zhang, Adv. Opt. Mater. 1, 186 (2013).

${ }^{8}$ C. Ma and Z. Liu, Appl. Phys. Lett. 96, 183103 (2010).

${ }^{9}$ Y. Huang, Q. Zhao, S. K. Kalyoncu, R. Torun, Y. Lu, F. Capolino, and O. Boyraz, Appl. Phys. Lett. 104, 161106 (2014).

${ }^{10}$ X. Wan, W. X. Jiang, H. F. Ma, and T. J. Cui, Appl. Phys. Lett. 104, 151601 (2014).

${ }^{11}$ C. Saeidi and D. van der Weide, Appl. Phys. Lett. 105, 053107 (2014).

${ }^{12}$ W. Wang, Z. Guo, R. Li, J. Zhang, Y. Li, Y. Liu, X. Wang, and S. Qu, Opt. Express 23, 16782 (2015).

${ }^{13}$ D. Lin, P. Fan, E. Hasman, and M. L. Brongersma, Science 345, 298 (2014).

${ }^{14}$ P. R. West, J. L. Stewart, A. V. Kildishev, V. M. Shalaev, V. V. Shkunov, F. Strohkendl, Y. A. Zakharenkov, R. K. Dodds, and R. Byren, Opt. Express 22, 26212 (2014).

${ }^{15}$ J. H. Lee, J. W. Yoon, M. J. Jung, J. K. Hong, S. H. Song, and R. Magnusson, Appl. Phys. Lett. 104, 233505 (2014).

${ }^{16}$ Y. Liu, X. Ling, X. Yi, X. Zhou, H. Luo, and S. Wen, Appl. Phys. Lett. 104, 191110 (2014).

${ }^{17}$ M. Decker, I. Staude, M. Falkner, J. Dominguez, D. N. Neshev, I. Brener, T. Pertsch, and Y. S. Kivshar, Adv. Opt. Mater. 3, 813 (2015).

${ }^{18}$ Y. F. Yu, A. Y. Zhu, R. Paniagua-Domínguez, Y. H. Fu, B. Luk'yanchuk, and A. I. Kuznetsov, Laser Photonics Rev. 9, 412 (2015).
${ }^{19}$ K. E. Chong, I. Staude, A. James, J. Dominguez, S. Liu, S. Campione, G. S. Subramania, T. S. Luk, M. Decker, D. N. Neshev et al., Nano Lett. 15, 5369 (2015).

${ }^{20}$ M. I. Shalaev, J. Sun, A. Tsukernik, A. Pandey, K. Nikolskiy, and N. M. Litchinitser, Nano Lett. 15, 6261 (2015).

${ }^{21}$ A. Arbabi, Y. Horie, A. J. Ball, M. Bagheri, and A. Faraon, Nat. Commun. 6, 7069 (2015).

${ }^{22}$ Y. Yang, I. I. Kravchenko, D. P. Briggs, and J. Valentine, Nat. Commun. 5, 5753 (2014).

${ }^{23}$ A. Arbabi, Y. Horie, M. Bagheri, and A. Faraon, Nat. Nanotechnol. 10, 937-943 (2015).

${ }^{24}$ S. W. Kim, K. J. Yee, M. Abashin, L. Pang, and Y. Fainman, Opt. Lett. 40, 2453 (2015).

${ }^{25}$ B. Desiatov, N. Mazurski, Y. Fainman, and U. Levy, Opt. Express 23, 22611 (2015).

${ }^{26}$ F. Aieta, M. A. Kats, P. Genevet, and F. Capasso, Science 347, 1342 (2015).

${ }^{27}$ A. F. Koenderink, A. Alù, and A. Polman, Science 348, 516 (2015).

${ }^{28}$ J. Kim, D. Shin, S. Choi, D.-S. Yoo, I. Seo, and K. Kim, Appl. Phys. Lett. 107, 101906 (2015).

${ }^{29}$ M. Khorasaninejad, W. T. Chen, R. C. Devlin, J. Oh, A. Y. Zhu, and F. Capasso, Science 352, 1190 (2016).

${ }^{30}$ M. Kerker, D.-S. Wang, and C. Giles, JOSA 73, 765 (1983). 